\title{
GPP
}

GESTIÓN Y ANÁLISIS DE POLÍTICAS PÚBLICAS, Nueva Época, n 13 enero-junio 2015 ISSN: 1989-8991

DOI: http://dx.doi.org/10.24965/gapp.voi13.10232

\section{La gestión integrada de recursos hídricos en la política federal del agua: propuesta para la nueva Ley General de Aguas en México}

\author{
Antonio Cañez Cota \\ Escuela de Gobierno del Tecnológico de Monterrey, México \\ tono_canez@hotmail.com
}

\section{Resumen}

El propósito del presente artículo es proponer un esbozo de política pública federal en el contexto del debate de la recién aprobada Ley General de Aguas en México. Se parte de un diagnóstico estadístico y se analiza el proceso de política pública desde el establecimiento de la agenda hasta la evaluación, pasando por la definición del problema, el diseño y la implementación. La lección del artículo es que los principales desafíos de la gestión integrada de recursos hídricos en México se encuentran a nivel local y es necesario que la política federal enfatice en el fortalecimiento de los organismos a nivel de cuenca y de municipio, se propone la gobernanza corporativa pública como una alternativa para el fortalecimiento institucional local.

Palabras clave

Política pública, gestión integrada de recursos hídricos, Ley General de Aguas, gobernanza corporativa pública, México.

\section{Integrated water resources management in the federal water policy: proposal for the new General Water Law in Mexico}

\begin{abstract}
The purpose of this study is to propose an outline for federal public policy in the context of the debate of the recently approved General Water Law in Mexico. It started from a statistical diagnosis and is analyzed the public policy process from agenda setting to evaluation, including the problem definition, design and implementation. The lessons of the article are that the main challenges of the integrated water resources management in Mexico are located locally and it is necessary that the federal policy emphasizes the strengthening of the organizations at basin and municipal level. It is put-forth that the public corporate governance can be an alternative to the local institutional strengthening.
\end{abstract}

Key words

public policy, integrated water resources management, General Water Law, public corporate governance, Mexico. 
El objetivo del presente estudio es analizar la gestión integrada de recursos hídricos en el contexto de la política federal del agua con el fin de proponer una estrategia complementaria para la implementación para la nueva Ley General de Aguas en México. El debate actual de este tema se encuentra en tránsito entre el Poder Legislativo y el poder Ejecutivo, el presente documento busca aportar una postura con ideas y evidencias que ayude a fortalecer la aplicación efectiva de la nueva legislación. La estructura del presente artículo se divide en cuatro partes. La primera, se refiere a una breve descripción de la organización del agua en México, con especial énfasis en lo relacionado a la nueva Ley General de Aguas. La segunda parte, muestra un diagnóstico que permite ubicar la problemática y situación de los recursos hídricos en el mundo y en México, se aportan datos y se muestra la complejidad y lo multifacético del tema del agua.

En la tercera parte, se analiza la política federal del agua como proceso, donde se incorpora lo siguiente: a) el establecimiento de la agenda, los actores que impulsan intereses transformados en definiciones de problemas y soluciones; b) la definición del problema de política pública con base en la visión de los actores expuestos en la agenda; c) el diseño de la política pública, se analizan las alternativas tradicionales contra una novedosa: la gestión integrada de recursos hídricos; d) los problemas previsibles de implementación, se pone sobre la mesa un mapa de actores e influencias para identificar los aliados y contrarios a la propuesta de la nueva legislación; por último e) la evaluación, en la que se evalúan las acciones de políticas y se proponen las estrategias para enmendar el camino cuando no se está cumpliendo con el núcleo duro del problema de política pública. En la cuarta y última parte del artículo, se presentan las conclusiones y se propone la gobernanza corporativa pública como una acción complementaria en el proceso de implementación con el fin de enfrentar efectivamente los desafíos de política pública en un contexto de la gran diversidad local en México.

\section{PARTE I}

\section{LA ORGANIZACIÓN DE LA GESTIÓN DEL AGUA EN MÉXICO}

En la idea de implementar la gobernanza del agua, las recomendaciones desde el Foro Mundial del Agua del año 2000, se han dirigido hacia un nuevo modelo de gestión llamado Gestión Integral de Recursos Hídricos (GIRH), que se enfoca en usar y conservar el agua a nivel de cuenca, así como promover una visión integradora y dejar atrás la visión sectorial, lo anterior bajo los principios de descentralización y participación. En México, a más de una década de implementación del modelo GIRH se han presentado problemas debido a que son varios los actores que confluyen en un territorio y ha faltado participación y consenso (cooperación) entre los diversos intereses individuales (CONAGUA, 2011).

La gestión integrada de recursos hídricos pretende integrar lo que está fragmentado, es decir, dirigir hacia objetivos comunes la acción de la variedad de organizaciones y grupos interesados en los asuntos del agua, integrar suelo y agua e integrar los beneficios económicos con los sociales y ambientales (ONU, 2008). Sin embargo, algunos críticos afirman que no hay evidencia empírica que demuestre que la GIRH funciona a nivel país o grandes cuencas hidrológicas, solo existe evidencia para afirmar que funciona a nivel local (Biswas, 2008).

En relación con el caso mexicano, la mayoría de las investigaciones coinciden en que el problema de la gestión del agua se encuentra en la debilidad de su marco institucional, la cual no ha permitido que la GIRH tenga los resultados esperados. Se implantó dicho modelo de gestión pero continúan las inercias y prácticas del pasado, es decir, la centralización del poder sigue imperando y no la descentralización y alta intensidad de participación de todos los actores como plantea el modelo (Domínguez, 2006; Nava y Sandoval, 2014). En este contexto se aprobó el dictamen de la Ley General de Aguas y es necesario que la política federal del agua se adapte a esta reforma.

La organización del agua en México se basa en trece regiones hidrológicas que son administradas por la Comisión Nacional del Agua (CONAGUA) a través de los Organismos de Cuenca y cuando la región abarca mucho territorio estos organismos se auxilian por direcciones locales para estar más cerca, territorialmente, de los grupos interesados. Estas organizaciones federales tienen facultades en todos los asuntos relacionados con el agua desde agua potable y saneamiento, concesiones de agua a particulares y asignaciones de agua a poblaciones, agua para la agricultura, hasta los aspectos geológicos y de protección de inundaciones como las grandes presas.

Cada Organismo de Cuenca atiende cuatro funciones básicas: a) Consejos de Cuenca, b) Distritos y Unidades de Riego, c) Agua Potable y Saneamiento, d) Asuntos Técnicos. El Consejo de Cuenca es la junta de varias organizaciones que reúnen a los grupos interesados para la elaboración de los planes a largo plazo, así como decidir las estrategias de remediación de problemas como inundaciones, sequias, problemas de contaminación de aguas,

1 La información se obtuvo del Reglamento de la Ley de Aguas Nacionales y del Reglamento Interior de la Comisión Nacional del Agua. Ambas disponibles en www.diputados.gob.mx 
entre otras. Los Consejos de Cuenca se sirven para sus propósitos de los Comités de Cuenca, Comités de Aguas Subterráneas, entre otros comités. Los Consejos de Cuenca son un instrumento importante de participación ciudadana en la gestión federal del agua en México.

En relación con los Distritos de Riego, son organizaciones que integran a los grandes agricultores que tienen alto consumo de agua en sus procesos productivos. Por su parte, las Unidades de Riego aglutinan a los pequeños agricultores que tienen bajo consumo de agua. Estos Distritos y Unidades de Riego se forman para aumentar la eficiencia en el uso de agua y la CONAGUA colabora con el 50\% del costo total de la rehabilitación de canales de riego y adquisición de tecnología que reduzca el consumo de agua en la agricultura, en ocasiones la Comisión Estatal del Agua (CEA) respectiva colabora con un monto adicional y la inversión es, en su mayoría, con presupuesto público.

Con relación en Agua Potable y Saneamiento, es un asunto de competencia básicamente municipal y la principal contribución federal por medio de CONAGUA se da con aportaciones del $50 \%$ del costo total de infraestructura. En los Organismos Operadores Municipales (OOM) existe un Consejo Consultivo que integra a funcionarios de la CONAGUA y CEA, así como a representantes del sector académico, empresarial y social. Estos OOM son descentralizados de la administración municipal lo que significa que tienen autonomía jurídica y financiera, sin embargo, el nombramiento del director general depende del Alcalde en turno y las finanzas dependen en gran medida de subsidios del presupuesto municipal ya que la mayoría de estos organismos están en crisis financiera debido a la predominancia de los intereses políticos sobre los intereses públicos en la toma de decisiones (Cáñez, 2011).

En el caso de las entidades federativas, cada una de ellas tiene una Comisión Estatal del Agua (CEA) que tiene como objetivo administrar los cuerpos de agua que no son competencia federal. Otra facultad relevante que tiene la CEA es prestar el servicio de agua potable en los municipios que no tienen la capacidad técnica y financiera. En este sentido, se observa un entramado organizacional disperso y plural en la organización del agua en México, sin embargo, la práctica real ha sido de centralización y esta variedad de organizaciones no ha tenido la influencia y el peso específico para influir en las decisiones principalmente en manos del poder ejecutivo, ya sea federal, estatal o municipal (Domínguez, 2010).

Además, México adoptó y reconoce la Gestión Integrada de Recursos Hídricos como su modelo de gestión y para contar con un modelo efectivo, además de organizaciones gubernamentales capaces, requiere de una ciudadanía en pleno ejercicio de sus derechos y obligaciones y en el caso mexicano se está lejos de contar con esas capacidades ciudadanas (Torregrosa et.al, 2012). El dictamen de la nueva Ley dice que la administración del agua en México requiere de la coordinación efectiva de los distintos órdenes de gobierno, del sector privado y social. El hacer realidad esta propuesta de cooperación parece ser el principal desafío de la gestión del agua en México.

En los artículos 33 al 36 del dictamen de la nueva Ley General de Aguas (2015) se contempla que los estados y municipios se coordinen para formar recursos humanos y profesionalizar el sector hídrico, sin embargo, la Ley parece quedar limitada porque solo se plasma como un buen deseo ya que no se establece quién es el responsable ni la forma de llevarlo a cabo. El dictamen de la nueva Ley se basa en la misma estructura de la casi abrogada Ley de Aguas Nacionales pero destacan un nuevo objetivo y un nuevo instrumento. El nuevo objetivo es asegurar el derecho humano al agua potable y saneamiento y el nuevo instrumento es la co-gestión del agua por medio de concesionar a privados el servicio en los municipios y generar asociaciones público privadas para infraestructura. Es en estos dos nuevos puntos donde el presente artículo pone énfasis.

El 5 de marzo de 2015 la cámara de diputados aprobó el dictamen de la nueva Ley General de Aguas con 31 votos a favor por los partidos PRI, PAN y PVEM, mientras que los diputados del PRD y MORENA² abandonaron la discusión ya que su minoría de votos no podía impedir la aprobación del dictamen y de inmediato iniciaron resistencias sociales, entre ellos la internacional Greenpeace, conglomerados de académicos especialistas en la materia y organizaciones de la sociedad civil. Básicamente argumentan que la nueva Ley no es específica en varios aspectos y eso abre la puerta a la privatización del agua, a promover procedimientos industriales que dañan el medio ambiente como el fracking. ${ }^{3}$

A partir de esta breve descripción de la organización del agua en México y de la transición hacia la nueva Ley General de Aguas, a continuación se presenta un diagnóstico estadístico con el objetivo de identificar los principales desafíos y problemas del agua en México para posteriormente hacer un análisis de política pública y estar en

2 PRI: Partido Revolucionario Institucional. PAN: Partido Acción Nacional. PRD: PVEM: Partido Verde Ecologista de México. PRD: Partido de la Revolución Democrática. MORENA: Partido del Movimiento de Regeneración Nacional.

3 La técnica del fracking es utilizada principalmente en la obtención del gas shale, es el proceso de fracturar la roca subterránea por medio de la inyección de agua a alta presión con el fin de liberar otra sustancia como petróleo o gas. (Oxford English Dictionary, consultado el 12 de abril de 2015 en: http://www.oxforddictionaries.com/definition/english/fracking). 
posición de proponer una estrategia complementaria para la implementación de la nueva Ley General de Aguas en México.

\section{PARTE II}

\section{EL DIAGNÓSTICO}

Cada vez existe mayor presión sobre los recursos hídricos en el mundo, el aumento de la población y de la actividad económica han demandado mayor cantidad de agua, por lo que en algunos países se ha extraído mayor cantidad de agua que la que tienen disponible en equilibrio, es decir, se extrae más agua de lo que ingresa a los acuíferos. A continuación se presenta un panorama para visualizar el problema a nivel país:

CUADRO 1: PAÍSES CON MAYOR GRADO DE PRESIÓN SOBRE LOS RECURSOS HÍDRICOS, 2010

\begin{tabular}{|l|l|r|r|r|}
\hline No. & \multicolumn{1}{|c|}{ País } & \multicolumn{1}{c|}{$\begin{array}{c}\text { Disponibilidad } \\
\left(\mathrm{km}^{3}\right)\end{array}$} & \multicolumn{1}{c|}{$\begin{array}{c}\text { Extracción total } \\
\left(\mathrm{km}^{3}\right)\end{array}$} & $\begin{array}{c}\text { Grado de presión sobre } \\
\text { los recursos hídricos (\%) }\end{array}$ \\
\hline 1 & Kuwait & 0.02 & 0.49 & 2465.00 \\
\hline 2 & Emiratos Árabes Unidos & 0.15 & 3.05 & 2032.00 \\
\hline 3 & Arabia Saudita & 2.40 & 22.64 & 943.30 \\
\hline 4 & Libia & 0.60 & 4.31 & 718.00 \\
\hline 20 & Irán & 137.50 & 93.10 & 67.71 \\
\hline 40 & Sudáfrica & 50.00 & 12.48 & 24.96 \\
\hline 52 & Turquía & 213.60 & 40.10 & 18.77 \\
\hline 53 & México & 462.58 & 80.30 & 17.36 \\
\hline 59 & Estados Unidos & 2818.00 & 477.80 & 15.57 \\
\hline 60 & Francia & 200.00 & 31.61 & 14.98 \\
\hline
\end{tabular}

Fuente: Atlas del Agua en México, Comisión Nacional del Agua, 2012.

Como se observa en el Cuadro 1, Kuwait es el país con mayor grado de presión sobre los recursos hídricos en el mundo, ya que extrae casi 24 veces más de lo que ingresa a los acuíferos por captación de lluvia, esto significa que el nivel de reserva histórico acumulado de los acuíferos disminuye año con año y de continuar esta tendencia desequilibrada de manejo de las aguas subterráneas dichos acuíferos podrían desaparecer y esos países tendrían que buscar fuentes de abastecimiento alternativas. Asimismo, Emiratos Árabes Unidos, Arabia Saudita y Libia tienen un grado de presión muy elevado. Un caso que salta a la vista es el de Estados Unidos, tiene una gran disponibilidad de agua por recarga de sus acuíferos y utiliza mucha agua, casi mil veces más de lo que utiliza Kuwait que es el país con mayor problema de presión hídrica. Por su parte, México tiene un grado de presión bajo sobre los recursos hídricos a nivel país, sin embargo, a continuación se muestra que al interior de los países hay diferencias regionales.

\section{CUADRO 2: CONTRASTE REGIONAL ENTRE DESARROLLO Y DISPONIBILIDAD DEL AGUA EN MÉXICO.}

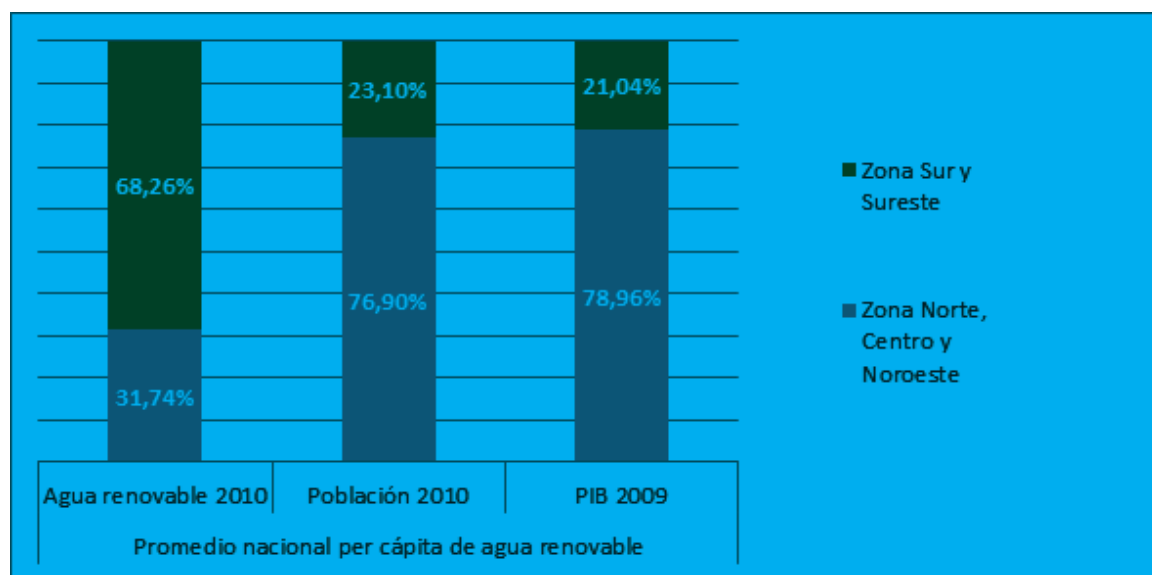

Fuente: Atlas del Agua en México 2012, Comisión Nacional del Agua, p. 18 
En el Cuadro 2, se observa que el uso intensivo del agua se lleva a cabo en la zona con menor disponibilidad, la zona norte, centro y noroeste cuenta con menos de un tercio del agua renovable del país y en esas zonas se concentra más de las tres cuartas partes de la población, así como también se produce más de las tres cuartas partes del Producto Interno Bruto (PIB) nacional. Debido a la diversidad de regiones y disponibilidad del agua del país es relevante ahondar en la disponibilidad de agua renovable per cápita por región hidrológico administrativa.

Se observa que las regiones con menores problemas de disponibilidad de agua son Frontera Sur y Golfo Centro, en cuanto a las regiones con mayores problemas son Aguas del Valle de México y Península de Baja California. Además, con el aumento de las sequías se agrava la disponibilidad en las zonas más áridas, así como en época de huracanes el exceso de agua hace que muchas personas pierdan su patrimonio en las zonas más húmedas. Cada temporada de huracanes deja daños humanos y materiales, principalmente en Pacífico Norte, Pacífico Sur y Frontera Sur. Asimismo, es relevante tomar en consideración la cantidad de agua renovable per cápita, se observa que en algunas regiones hay desproporción entre el agua utilizada y la que se renueva, a continuación se muestra este aspecto (Conagua, 2012).

Hay regiones en México con un alto nivel de presión sobre los recursos hídricos, la región Lerma-SantiagoPacífico por sí sola tiene casi un tercio de los acuíferos sobreexplotados del país, si se le suman las regiones de Cuencas Centrales del Norte y Río Bravo solo estas tres regiones de las trece totales tienen más de las dos terceras partes de los acuíferos sobreexplotados. Una explicación es la actividad agrícola de estas regiones, ya que ésta se lleva más de las tres cuartas partes del uso total del agua en México. Este punto es central en el análisis de la política y gestión del agua ya que uno de los problemas más recurrentes es el conflicto entre el uso del agua agrícola y el uso de agua para las ciudades (Semarnat, 2012).

CUADRo 3: usos CONSUNTIVOS DEL AGUA EN México: origen y volumen, 2010

\begin{tabular}{|l|c|c|c|c|}
\hline \multirow{2}{*}{ Uso } & \multicolumn{2}{|c|}{ Origen } & Volumen total en super- & $\begin{array}{c}\text { Porcentaje de } \\
\text { extracción (\%) }\end{array}$ \\
\cline { 2 - 5 } & Superficial & Subterráneo & 61.49 & 76.7 \\
\hline Agrícola & 40.57 & 20.92 & & 11.44 \\
\hline Abastecimiento público & 4.33 & 7.11 & & 4.2 \\
\hline Industria autoabastecida & 1.47 & 1.73 & 3.20 & 4.0 \\
\hline $\begin{array}{l}\text { Energía eléctrica exclu- } \\
\text { yendo hidroelectricidad }\end{array}$ & 3.63 & 0.44 & 4.08 & 5.1 \\
\hline Total & 50.01 & 30.20 & 80.21 & 100.00 \\
\hline
\end{tabular}

Fuente: Estadísticas del Agua en México 2012, pág. 36.

En el Cuadro 3, se observa que el uso que mayor cantidad de agua consume en México es la agricultura, seguido por el abastecimiento público urbano y por el industrial. Otro aspecto importante es que las ciudades y la industria dependen en mayor medida del agua subterránea. Por su parte, a pesar de que la agricultura utiliza más el agua superficial es el mayor consumidor de agua subterránea, especialmente existe un uso intensivo de agua subterránea en la agricultura en las zonas con mayor escasez del vital líquido (Conagua, 2012). Este diagnóstico no estaría completo sin el análisis de la cobertura de agua potable y saneamiento.

En los municipios mexicanos se observa una cobertura ligeramente mayor para agua potable que para saneamiento, se destaca la relación entre baja disponibilidad de agua y alta cobertura de agua potable y saneamiento, así como alta disponibilidad de agua y baja cobertura. En el caso de las regiones hidrológicas de Pacífico Sur y Frontera Sur, que son las regiones con mayor cantidad de agua disponible, son las que cuentan con menor cobertura de agua potable y saneamiento en México (Conagua, 2012). Este dato indica es una muestra de que los problemas del agua van más allá de un asunto de escasez o abundancia y que se requiere enfocarse en la política y gestión del agua.

En resumen, los datos presentados en este diagnóstico arrojan problemas de acuíferos sobreexplotados, baja eficiencia en el uso de agua en la agricultura, regiones mexicanas con mayor disponibilidad de agua que otras y, sin embargo, las regiones con más agua son las que tienen la menor cobertura de agua potable y saneamiento. Este 
último dato lleva a la necesidad de pensar el problema de la política del agua no como un problema técnico o de disponibilidad sino como un problema de gestión y del marco institucional (Domínguez, 2006). La gran fotografía producto de los datos muestra que hay grandes disparidades regionales y el tratar de integrar estas diferencias representa un gran desafío para la gestión y política federal del agua en México. A continuación, se presenta un análisis de política pública con el fin de contextualizar la gestión integrada de recursos hídricos dentro de la política federal.

\section{PARTE III}

\section{ANÁLISIS DE POLÍTICA PÚBLICA}

Adaptar la generalidad de la política federal del agua con las especificidades locales es un gran desafío y es necesario que la política establezca el paraguas general y el mínimo indispensable para la integración del manejo del agua a nivel país. A continuación, se muestra un análisis de política pública con el fin de identificar los problemas de política hídrica y en la siguiente parte de este artículo finalizar con una propuesta de estrategia para la nueva Ley General de Aguas.

\section{El establecimiento de la agenda}

Se identifican seis actores con capacidad de influencia en la política federal del agua en el contexto de la aprobación de la nueva Lay General de Aguas en México, estos actores son: la Comisión Nacional del Agua, los Gobernadores de los Estados, los grandes agricultores organizados en Distritos de Riego, la Asociación Nacional de Empresas de Agua Potable y Saneamiento y los Legisladores integrantes de las Comisiones relacionadas con el agua. Los actores con mayor capacidad de influencia son:

CUADRO 4: ANÁLISIS DE LA INFLUENCIA DE LOS ACTORES EN EL ESTABLECIMIENTO DE LA AGENDA DE POLÍTICA PÚBLICA FEDERAL DEL AGUA EN MÉXICO

\begin{tabular}{|c|c|}
\hline Actor & Cómo define el problema \\
\hline Comisión Nacional del Agua & Metas formales del Gobierno Federal \\
\hline $\begin{array}{c}\text { Asociación Nacional de Empresas de } \\
\text { Agua Potable y Saneamiento }\end{array}$ & Infraestructura e Insumos \\
\hline Legisladores Federales & Organización de la Gestión en Ley \\
\hline Sector Agrícola & Mantener y ampliar subsidios y beneficios para producción de \\
alimentos
\end{tabular}

Fuente: elaboración propia con base en la bibliografía del presente documento, incluyendo el análisis de las noticias para conocer la posición de los actores con respecto al tema del agua.

El Cuadro 4, muestra que el establecimiento de la agenda está íntimamente ligado a la definición del problema y a las soluciones, sin embargo, estas partes se separan como parte del proceso de políticas públicas con fines analíticos (Aguilar, 1993b). Para hacer el análisis del establecimiento de la agenda, se toman en cuenta los actores del cuadro 4 y tres tipos de agendas: pública (temas que la ciudadanía intenta impulsar para que los representantes los tomen y los lleven a la agenda política y después a la gubernamental), política (temas que alcanzan prioridad en el debate y acción de los políticos y representantes, están en el debate político y legislativo) y gubernamental (temas que el gobierno planea en forma de proyecto y asigna recursos públicos). En los sistemas democráticos se busca que la relación entre las tres agendas sea estrecha como producto del tratamiento de temas que a la ciudadanía le interesa (Casar y Maldonado, 2010).

En relación con la agenda pública, se observa una mayor participación de la Comisión Nacional del Agua (CONAGUA) y de los Gobernadores de los Estados. Ambos, definen el problema como de acceso al agua potable y saneamiento para uso público urbano, mayor productividad del agua en el sector productivo, y en estados afectados por huracanes incluyen las obras de control de inundaciones. ${ }^{4}$ Queda rezagado el tema de la contaminación de los cuerpos de agua. En lo que respecta a la agenda política, se aprobó el dictamen para la creación de la Ley

4 Análisis realizado en google noticias con el criterio de número de veces de aparición del tema por parte de cada actor, consultado el 19 de noviembre de 2013 en http://news.google.com.mx/ 
General de Aguas pero se estancó su aprobación definitiva por fuertes presiones de grupos de la sociedad civil y de académicos especialistas en el tema del agua. Este conflicto es muestra de que el tratamiento de la agenda no fue incluyente.

Con relación en la agenda gubernamental, actualmente el problema está definido como la falta de infraestructura ya que la estrategia de los programas de CONAGUA es incentivar la creación y rehabilitación de infraestructura y el mayor contrapeso efectivo que tiene la CONAGUA son los gobernadores de los estados y éstos impulsan el tema del agua como infraestructura. A pesar de que México adoptó el modelo de gestión integrada de recursos hídricos el desafío principal es cómo aplicar efectivamente este modelo que tiene alrededor de 10 años en México y aún no se consolida su marco institucional, es decir, la desconcentración del poder y la integración efectiva de los actores públicos, privados y sociales (Domínguez, 2006).

El conflicto en la conformación de prioridades en la agenda mexicana gira en torno a tres conflictos históricos. Primero, se encuentra el conflicto por los usos del agua y la sobre explotación de los mantos acuíferos por parte de los agricultores, para los cuales el agua superficial no tiene costo, solo tiene un costo indirecto cuando el agua es subterránea y se requiere energía eléctrica para el bombeo. En esta área se presenta el conflicto latente entre el uso público urbano y el uso agrícola, por una parte, los organismos operadores municipales de agua potable afirman que los agricultores utilizan la mayor cantidad de agua y desperdician mucha por los sistemas de riego ineficientes; por otra parte, los agricultores argumentan que tienen concesionado cierto volumen de agua y la extracción para consumo ajeno al agrícola puede poner en riesgo la disponibilidad para el riego y, por lo tanto, el incremento en los precios de los alimentos. ${ }^{5}$ En este sentido, hay dos temas en conflicto en la conformación de la agenda: uso para consumo humano contra uso para la agricultura.

Segundo, se encuentra el conflicto por los daños causados por los fenómenos hidrometeorológicos, en la temporada de huracanes aparece el tema en la agenda e incluso se ha formado un fondo para la atención de desastres naturales. La temporalidad de los huracanes, meses de septiembre y octubre, beneficia al tema de emergencias e inundaciones porque, cuando está el tema vigente en los medios de comunicación, el tema es muy sensible porque son las mismas fechas que las negociaciones del presupuesto, así que el tema tiene una temporalidad en favor de captar recursos públicos presupuestales. El tema en la agenda de inundaciones se da por la afectación a la economía y patrimonio de miles de familias y negocios cada año. El tema de mayores recursos para atención de desastres naturales es apoyado por los gobernantes de todos los órdenes de gobierno porque significa la ejecución de más infraestructura

Por último, el conflicto por la contaminación del agua y el tratamiento de aguas residuales. Es el conflicto que tiene menor resonancia en los medios de comunicación y en la deliberación pública, el utilizar y tratar las aguas de manera correcta representa mayores costos para los industriales, agricultores y organismos operadores municipales, lo que sumado a una baja capacidad de enforcement ${ }^{6}$, provoca que dicho tema pase desapercibido. Los organismos internacionales han estado empujando este tema en la agenda pero sin un impacto significativo en la atención oficial en México. Este tema recién entro en la agenda no por voluntad de los actores sino por una ventana de oportunidad (Kingdon, 1984), el 6 de agosto de 2014 ocurrió el peor desastre natural por contaminación de un rio en México, se derramaron más de 40 mil metros cúbicos de ácido sulfúrico en el Rio Sonora y dejo afectacio-

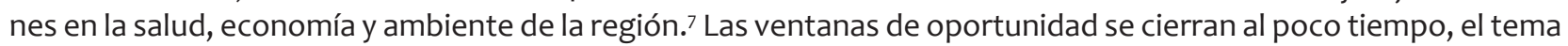
es ahora sensible en la agenda pública pero requiere que la integración de las acciones sea efectiva y se transite de una gestión federal basada en infraestructura a una gestión integrada de recursos hídricos basada en consensos.

En resumen, en la conformación de la agenda no se involucran "temas" en abstracto, sino que se imponen y pugnan definiciones de problemas para ganar la batalla de obtener visibilidad y recursos públicos. En sí, se trata de un paquete de definiciones de problemas y soluciones (Bardach, 2000). Los actores empujan temas a la agenda con el fin de obtener ganancias, ya sea material, política, económica e, incluso ideológica. Es este precisamente uno de los grandes desafíos del diseño de políticas públicas: el desafío político y social que tiene que enfrentar cualquier política. Debido a ello es trascendental definir claramente el problema que atiende la política pública (Majone, 1997).

5 El último caso de impacto nacional es el conflicto entre el Gobierno del Estado de Sonora y los agricultores del Valle del Yaqui, quienes alegan que es ilegal el Acueducto Independencia que dota de agua a la ciudad de Hermosillo, Sonora.

6 Enforcement lo entiendo como capacidad de asegurar el cumplimiento de una norma.

$7 \quad$ Nota muy difundida en varios medios impresos y electrónicos nacionales e internacionales, para muestra véase: http://internacional.elpais.com/internacional/2014/08/27/actualidad/1409095702_528258.html 


\section{La definición del problema}

Es importante señalar que la definición del problema viene con una carga de contenido importante desde la agenda, en la manera en cómo los actores calificaron y moldearon el tema, le dieron sustancia de causalidad y de solución (Aguilar, 1993C). Los problemas públicos tienen mucho que ver con las valoraciones de la gente y el contexto, lo que es un problema de escasez de agua aquí es una problema de inundación allá, por una parte, un problema de contaminación de algún río y, por otra parte, un problema de desperdicio de agua por fugas en la red de agua potable o en los canales de riego. Si bien es cierto que la definición de problema se construye por las valoraciones de los actores, ¿cómo definir un problema público desde un punto de vista académico? ¿Cómo definir un problema tan escurridizo como el agua? ¿Es deseable descomponer el problema en varios problemas menores? ¿Cómo definir un problema general y nacional ante tal variedad de temas, dimensiones y regiones del agua en México?

Estas preguntas son más para ubicar la dimensión de complejidad de una política nacional del agua que para dar respuestas definitivas, sin embargo, se presenta una reflexión a estas interrogantes (Heylighen, 1999) Es indispensable pensar en la política pública nacional del agua que coordine las distintas acciones con una visión y un valor de referencia que aglutine los esfuerzos y recursos en una dirección y le otorgue un sentido, esto se refiere a la racionalidad de las políticas públicas, a pensar los problemas como causalidad, a medirlos para manejarlos. La dificultad de definir el problema como causa-efecto es que se corre el riesgo de caer en una hipótesis incorrecta, pero apenas así se acerca la definición correcta de un problema púbico (Aguilar, 1993a).

Las definiciones de problema de los actores interesados buscarán llegar en alguna parte del proceso de la política pública, de los programas y presupuestos, sin embargo, el actor que tienen la responsabilidad de otorgar sentido a la política federal del agua es la CONAGUA, el problema debe ser definido en una dimensión integrada y enfocarse en fortalecer el marco institucional de la gestión. En este sentido, este documento considera que, con base en la visión de la CONAGUA, el problema público que atiende la política federal del agua es que:

“Hay personas en México para quienes el agua potable y saneamiento es inasequible, el agua para uso productivo inaccesible y que pierden patrimonio por inundaciones. Además, la sobreexplotación y contaminación de cuerpos de agua pone en riesgo a los seres vivos."

Definir en una frase el problema público es una de las etapas que requiere mayor análisis y ejercicio intelectual en el proceso del análisis de política pública. Es decir, no cualquier problema debe ser público, tal como lo dice el padre fundador del estudio de las políticas públicas Harold Lasswell (1993): las políticas públicas son orientadas a los problemas fundamentales del ser humano en sociedad democrática, tienen un compromiso humanista y contextual.

En toda política pública se debe cuestionar la intervención del gobierno, ¿por qué el gobierno debe invertir recursos públicos en este problema del agua y no en otros sectores? Ninguna política puede aspirar a la neutralidad ética, "desprovisto de todo contenido ético el enfoque de política pública no solo puede perder profundidad sino que minaría incluso su eficacia. En el extremo podría convertirse en un asunto de técnicos preparados acaso para realizar estudios limitados, a la luz de datos inevitablemente parciales."(Merino, 2010:27). Con base en lo anterior, la intervención gubernamental, en el problema del agua que se plantea, es porque está ausente el valor de la integración producto de la debilidad institucional.

Es claro que la ausencia del valor de la integración no es evidente para todos y algunos podrían construir el problema de otra manera, sin embargo, es importante destacar que la política federal debe velar por los temas que constitucionalmente tiene a su cargo aunque la implementación final dependa de un entramado complejo de organizaciones, en este sentido la política federal del agua debe velar por integrar los siguientes elementos: a) es injusto que haya personas que no tengan asequibilidad de agua potable y saneamiento; b) es injusto que haya personas que no tengan acceso al agua para desarrollar actividades productivas; c) es injusto que haya personas que pierdan patrimonio por inundaciones; d) es injusto que se contamine el agua porque disminuye la calidad de vida de seres vivos actuales y futuros.

El valor de la integración en la definición del problema es lo que debe guiar las decisiones en la política del agua en México, si bien es cierto que algunos investigadores consideran que el valor debe ser la eficiencia, hay que distinguir que la eficiencia es necesaria pero no suficiente, es decir, la eficiencia se requiere para no desperdiciar el recurso pero la integración se requiere para evitar que unos tengan en exceso y otros no tengan ni para tomar, en este sentido el valor de la integración como inclusión. 
La definición de un problema público tiene detrás una meta política (Parsons, 2007) o teoría de entrada (Merino, 2010), es decir, una teoría que explica y enfoca la atención en ciertos elementos, al mismo tiempo, que excluye otros. Por ejemplo, un mismo problema se puede definir como fallas de mercado, fallas de gobierno, perspectiva organizacional, análisis político estratégico, elección racional, entre otros (Weimer y Vining, 2002). Debido a la diversidad de enfoques, el analista de políticas públicas debe explicitar su teoría de entrada al momento de definir el problema. En la política federal del agua en México, la metapolítica o teoría de entrada es el análisis organizacional enfocado a la implementación ya que el objetico del presente análisis de política pública es proponer una estrategia complementaria para la puesta en marcha de la Nueva Ley General de Aguas. La teoría de entrada es la necesidad de ligar la política federal del agua con las políticas locales y con la gestión integrada de recursos hídricos, es por ello la integración.

En resumen, la definición de problema de política pública hídrica nacional supone la corresponsabilidad de los tres órdenes de gobierno y la sociedad en general. Por ello, es necesario ubicar el supuesto en el que descansa la política del agua en México, múltiples involucrados en la gestión: gobierno federal, gobiernos estatales, gobiernos municipales y participación privada y ciudadana.

\section{El diseño de la política pública}

El diseño o hechura de las políticas públicas se encuentra en el centro de la tensión entre dos posturas: por un lado, lo que podría representar Yazehkel Dror (1983), la de los que tratan de dotar a las políticas del conocimiento científico. Por otro lado, los que podría encabezar Charles Lindblom (1993), la de los que tratan de destacar el conflicto político y de negociación entre actores en las políticas.

Por el lado de los científicos, la tesis principal de Dror (1983) es que hay una brecha significativa entre las maneras en que individuos e instituciones hacen la política pública y el conocimiento disponible en cómo la política pública pudiera ser hecha de mejor manera. Es decir, la brecha entre el ser y el deber ser, entre la manera como realmente sucede y la manera que debería ser. Por el lado de los que ven la negociación política como lo que marca a la hechura de políticas, la tesis principal de Charles Lindblom (1993) es que las políticas se hacen de manera incremental, paso a paso, por medio de negociaciones y ajustes al margen. Este enfoque supone que la acción no es la racional tradicional sino la racional estratégica. A continuación se muestran las alternativas de la teoría causal del diseño de política pública del agua en México:

Las alternativas de solución al problema público son: a) el problema como falla de mercado: se llamará (gobierno); b) el problema de gobierno: se llamará (mercado) y; c) problema de cooperación: se llamará (integración). A continuación, con base en Morse y Struyk (2006), se presenta un cuadro que resume las alternativas de solución, costos, beneficios y factibilidad de las alternativas de solución.

CUADRO 5.- ANÁLISIS DE ALTERNATIVAS DE POLÍTICA PÚBLICA

\begin{tabular}{|c|c|c|c|c|}
\hline Alternativa & Costos & Factibilidad Política & Factibilidad Administrativa & Factibilidad Jurídica \\
\hline Gobierno & 3 & 3 & 3 & 2 \\
\hline Mercado & 2 & 1 & 1 & 1 \\
\hline Integración & 1 & 2 & 2 & 3 \\
\hline
\end{tabular}
intensidad de costo, beneficio y factibilidad.

En relación con el cuadro $5,{ }^{8}$ se observa que los menores costos concentrados los tiene Integración, ya que al ser un esquema cooperativo los costos se distribuyen en varios actores. La alternativa más costosa es Por Gobierno, esto debido a que se repiten tareas y obras, además, la falta de coordinación de las acciones aumenta los costos. En lo que concierne a la mayor factibilidad política la tiene Gobierno, porque los actores políticos muestran sus resultados a sus electores y obtienen mayores beneficios en las obras de infraestructura; por su parte, la menor factibilidad política la tiene Mercado debido a que en México se tiene desconfianza en la intervención del sector privado en los asuntos públicos.

La menor factibilidad administrativa la tiene Integración, ya que supone un nuevo modelo de gestión con base en la participación de actores públicos, sociales y privados en la gestión del agua, como México ha sido un país en el que el gobierno ha dirigido e implementado las políticas públicas, los actores involucrados no tienen la capaci-

8 El análisis de alternativas se llevó a cabo con los criterios del enfoque de política pública y de la sensibilidad propia del autor, cabe señalar que el presente documento es una invitación para abrir el debate administrativo de la nueva Ley General de Aguas en México. 
dad institucional de gestionar a través de redes. La mayor factibilidad administrativa la tiene Gobierno porque es la manera actual de operar y hay muchos incentivos productos de la generación de obra pública.

En cuanto a la factibilidad jurídica, Integración la tiene mayor porque el dictamen de la Nueva Ley General de Aguas propone una gestión integrada entre las dependencias públicas involucradas en todos los niveles de gobierno y con la participación del sector público, privado y social. En este sentido, la factibilidad jurídica es muy alta, falta una estrategia para fortalecer las capacidades institucionales de los actores para lograr la integración. La factibilidad jurídica más débil es Mercado, ya que si bien el dictamen de la nueva Ley toma en cuenta este tipo de instrumentos en México existen fuertes resistencias culturales.

Un elemento básico que se debe tomar en cuenta en el diseño de políticas es el elemento cultural. De acuerdo con Stephen Linders y Guy Peters (1993), hay fallas en la formulación de alternativas cuando no se toman en cuenta los instrumentos y las instituciones en las cuales se va a desarrollar la política pública, es decir, cuando no se toma en cuenta el contexto de aplicación y surgen problemas se debe al diseño y no a la implementación. En relación con las alternativas, Gobierno es la que tiene mayor aceptación cultural debido a que la gestión del agua ha sido de esta manera, sin embargo, el discurso de todos los actores reconoce que la Integración es necesaria para resolver los problemas del agua.

Decidirse por una alternativa requiere estar consciente de los costos y factibilidades, pero también requiere estar alertar para identificar cuándo lo factible puede llevar a costos elevados en el futuro. En el caso de la gestión del agua en México, es urgente transitar hacia el fortalecimiento de los entes locales, ya que el gobierno federal invierte cada año más recursos y los entes locales no lo hacen en igual proporción. Si revienta el problema, al mismo tiempo en varias regiones del país, no habrá dinero que alcance. Es necesario ubicar que el acceso asequible al agua potable y saneamiento se refiere a llevar a los que no tienen pero también a mantener la red ya existente y eso es muy costoso y prácticamente inviable si se pretende dirigir por Gobierno desde el centro y solo con infraestructura, la cual ha sido la única estrategia en varias décadas.

La capacidad institucional del país no está acorde con los retos de Integración en materia de agua, los entes locales no tienen finanzas sanas, los aqueja la corrupción, hay mucha rotación de personal, poca profesionalización y ausencia de proyectos a largo plazo. El nuevo diseño institucional en materia de agua supone la gestión integrada de recursos hídricos, entonces la política hídrica nacional debe enfocar sus acciones a coordinar esfuerzos, diseñar incentivos y fortalecer las capacidades institucionales de los actores locales para alcanzar resultados públicos efectivos, ya que la gestión del agua se da en lo local, en la cuenca, en el territorio, con la costumbre e historia de la gente (Domínguez, 2006).

Después de revisar las alternativas de política pública se obtiene que la mejor opción es el modelo de integración para la gestión integrada de recursos hídricos. Es la alternativa que debe conducir la política federal del agua en México y, a partir de ahí, diseñar los incentivos del marco institucional para conducir los ámbitos de competencia de los gobiernos estatales, municipales, actores sociales y privados, sobre todo, trabajar en incrementar las capacidades gerenciales de los actores involucrados para poder conectarse entre ellos y dirigirse hacia objetivos comunes.

En este sentido, la nueva Ley General de Aguas tiene un diseño de co-participación, de redes de implementación, de coordinación entre órdenes de gobierno y espacios para la participación privada y social. Es necesario llamar la atención sobre un aspecto central: el diseño requiere tomar en cuenta la dimensión del poder. Pensar la estructura institucional en función de los intereses y del poder de los actores, tratar de anticiparse a los incentivos que producirán las nuevas reglas del juego. A su vez, tomar en cuenta que el cambiar la regla formal no necesariamente cambia la regla informal ni la estructura del juego (Crozier y Friedberg, 1990). A continuación se analiza la fase de la implementación de política pública.

\section{La implementación}

De acuerdo con Robert Behn (1993), se debe tomar en cuenta el papel de los actores para hacer un mapa de influencias. A los analistas de políticas les interesa la eficiencia económica y conocer cómo las políticas pueden afectar a la sociedad en su conjunto. Asimismo, los analistas buscan medir con claridad los resultados de la política y el grado en que se realizan los objetivos. En contraste, a los políticos les interesa la distribución de los productos, y cómo las políticas públicas afectan a ciertos individuos y grupos. Ponen el acento en la negociación, en la transacción y el compromiso, tratan de cancelar los objetivos que impiden llegar a un consenso y buscan resolver el conflicto entre intereses rivales, distribuyendo recursos limitados. 
El éxito de la política pública depende, en gran medida, de la negociación con los actores involucrados, no se trata de quitarle los beneficios sino de tener un mejor manejo integrado de recursos hídricos, entender que la falta de atención y seguimiento a este tema puede perjudicar la disponibilidad, salud y bienestar de la población en su conjunto. De acuerdo con Eugene Bardach (1977), a pesar de que una política pública cuente con el mejor diseño y con el mejor ambiente de apoyo, puede llegar a fracasar si no tiene en cuenta la relevancia de su modo de ejecución. Este autor propone que la participación de distintos actores en la red de implementación puede verse como un juego, como el deseo de los participantes de ganar posiciones en una política pública. Estos juegos son problemas de implementación que ocurren sistemáticamente en las políticas públicas en Estados Unidos. Ahora bien, ¿cómo ocurren éstos problemas de implementación en México?

El juego de implementación clásico en México y en el que se debe poner especial atención es el de "estar disponible" (Bardach, 1977), se da cuando una política pública es capturada por algún partido o grupo político para beneficiar a su clientela electoral, ésta es una situación difícil porque no necesariamente se puede comprobar y sancionar legalmente. Estos problemas de implementación arrojan un elemento en común: la política pública se implementa en una organización compuesta por actores, los cuales pueden anteponer sus propios intereses a los de la política. La corrupción es un fuerte obstáculo a la eficacia de las políticas públicas del agua ya que es un sector que requiere grandes inversiones en infraestructura y los actores buscan obtener beneficios de las obras (Transparencia internacional, 2008).

De acuerdo con Pressman y Wildavsky (1984), hay dos conclusiones en relación con el carácter político de la implementación: la primera es que no hay ciencia que llegue al punto último de verdad, y segundo que la centralización del poder no sólo es más improductiva sino que también es más vulnerable a la captura privada de objetivos públicos, ya que la concentración de poder lleva a la imposición de unos valores sobre otros. En este sentido, la política federal del agua inició mal desde la aprobación del dictamen de la nueva Ley ya que no hubo la inclusión suficiente de actores interesados, el éxito en la implementación de la estrategia de Integración depende del fortalecimiento de las capacidades de los actores locales. A continuación, se presenta la fase de evaluación de la política pública.

\section{La evaluación}

La evaluación de una política pública es, como bien anota Mauricio Merino (2009:15), "la apreciación, la medición y el cálculo que cada política pública tiene, a la luz de los problemas públicos que quiere resolver, de las causas que ha definido, de los cauces factibles que estableció y de los valores que cada una invocó." Otro enfoque de evaluación es la medición de impacto, aislar el efecto de cómo las acciones de la política pública arrojaron resultados para cambiar la situación inicial que la política pública planteaba (Parsons, 2007), pero en este trabajo no se profundizará en este enfoque sino en el básico que tiene que ver con la valoración de los resultados de política con la definición del problema y los objetivos propuestos en el diseño.

Se debe destacar que la evaluación no necesariamente se realiza mucho tiempo después de la implementación, es necesario hacer una serie de evaluaciones con distinta temporalidad para valorar distintos aspectos de la política pública. Las evaluaciones realizadas al poco tiempo buscan mejorar el proceso de implementación mientras que las que se llevan a cabo un tiempo considerable después buscan retroalimentar la definición del problema, el diseño, los resultados y el impacto de las políticas (Parsons, 2007). El éxito de la política nacional del agua en el contexto de la nueva Ley General de Aguas depende, en gran medida, de los incentivos que genere el diseño institucional, y para ello, el contar con una participación efectiva y transparente de todos los actores involucrados aumentará la probabilidad de alcanzar los objetivos, así como tener procesos de innovación para retroalimentar y ajustar el proceso de política pública.

La evaluación tiene relación íntima con la definición del problema, es necesario definir claramente el problema para tener mayor probabilidad de éxito en la implementación y evaluación. La definición del problema de la política federal del agua es compleja porque requiere cuidar la disponibilidad del agua, calidad, cobertura universal, utilización agrícola, ecológica e industrial, eficiencia, equidad social, tratamiento y reutilización. El valor de la política pública es Integración y requiere que el modelo de gestión integrada de recursos hídricos sea efectivo en lograr la integración de todos los usos del agua y de todos los intereses de los actores involucrados, por ejemplo, integrar la competitividad del sector agrícola con la garantía del derecho humano al agua potable y saneamiento.

En el nuevo modelo de política pública que plantea la nueva Ley General de Aguas se requiere involucrar a los mismos agentes que participan en la implementación e incluso a otros agentes interesados para contar con contrapesos entre los distintos intereses, porque ello aumenta la probabilidad de eficacia y compromiso para las 
otras fases del proceso. El modelo de evaluación que requiere la nueva política federal del agua es un modelo descentralizado cercano a los actores, este modelo más enfocado a procesos de aprendizaje y mejora que enfocado a cuestiones burocráticas de control. La evaluación hace un recordatorio a los actores del núcleo duro de la política pública que persiguen y es necesario que la política cuente con un modelo de gestión efectivo. A continuación, se presenta una propuesta de estrategia que complemente la implementación del modelo de gestión integrada de recursos hídricos en México.

\section{PARTE IV}

\section{LA GOBERNANZA CORPORATIVA PUBLICA COMO INSTRUMENTO DE CONEXIÓN EFECTIVA ENTRE LA POLÍTICA FEDERAL DEL AGUA Y LA GESTIÓN INTEGRADA DE RECURSOS HÍDRICOS}

Es un hecho que a nivel mundial la gobernanza burocrática tradicional ha evolucionado a formas de organización más abiertas que suponen intercambios y cogestión entre actores gubernamentales, privados y sociales; la burocracia ya no es el único actor que participa en el proceso de toma de decisiones públicas. A esta evolución se le ha llamado: la Nueva Gobernanza Democrática. Algunos pueden acusar al Modelo Weberiano de los problemas de ineficacia del gobierno y pueden ver a la nueva gobernanza como la gran solución. Si bien es cierto que es un hecho la evolución histórica, el cambio de gobernanza (proceso de gobernar) no resolverá por sí solo los problemas que no pudo resolver la gobernanza tradicional. De hecho, es importante tener en cuenta los problemas tradicionales que no han sido solucionados y que la nueva gobernanza enfrentará: insuficiencia en la capacidad del gobierno para dar resultados públicos (Aguilar, 2013a).

En un estado democrático y federal, con un discurso de nueva gobernanza democrática y bajo un modelo de gestión integrada de recursos hídricos, interactúan varios actores (gubernamentales, privados y sociales) y varios marcos normativos (federal, estatal y municipal); se requiere encontrar la manera para que la dirección de las organizaciones, redes y políticas públicas multinivel y multipropósito sea eficaz y de resultados públicos. Uno de los principales desafíos del cambio de modo de gobernanza es que se requiere fortalecer las capacidades no solo gubernamentales sino que se requieren también ciudadanos en pleno ejercicio de sus derechos y obligaciones y aun México está lejos de contar con esas capacidades ciudadanas (Torregrosa et.al., 2012).

El problema con los instrumentos de participación en contextos de debilidad institucional es que solo sirven para legitimar el statu quo sin poner a disposición del ciudadano verdaderos espacios de participación y de poder para influir en los asuntos públicos (Ruano, 2010). En este sentido, la gestión integrada de recursos hídricos pretende integrar lo que está fragmentado y requiere que cada uno de los nodos de participación tenga la capacidad de conectarse con la red para alcanzar objetivos comunes de política pública porque si existen nodos sin la capacidad suficiente los objetivos serán menos públicos en el sentido de incluyentes y democráticos. Esta capacidad conectiva (Edelenbos et. al., 2013) es lo que requiere actualmente la GIRH para tener una política pública federal efectiva.

En este sentido, la efectividad de la política federal y la pertinencia del modelo GIRH dependen de las capacidades de los nodos de la red. Con base en lo anterior, la propuesta para la nueva Ley General de Aguas es impulsar una estrategia de gobernanza corporativa publica (Aguilar, 2013b) enfocado en la alta dirección pública de las organizaciones y en la conformación de las distintas juntas que tratan el tema del agua. Desde los Consejos de Cuenca a nivel de cuenca hidrológica hasta los Consejos Consultivos de los Organismos Operadores Municipales. Si bien es cierto que un cambio institucional requiere un cambio cultural, la estrategia para hacer efectiva la integración debe iniciar con los consejos directivos que integran a las organizaciones, a partir de ahí se despliega el cambio institucional al interior de estas organizaciones.

El Programa Nacional Hídrico (2014) contempla vagamente el fortalecimiento de los recursos humanos en el sector hídrico mexicano pero a la mitad del gobierno federal actual no se ha impulsado una estrategia para ello y la idea está más enfocada hacia lo técnico que hacia lo gerencial. Si se desea obtener una GIRH efectiva en el terreno de la implementación se deben buscar las mejores formas de generar capacidad gerencial en la administración pública. De acuerdo con Luis F. Aguilar (2013b), la Gobernanza Corporativa Publica es el eslabón que hace falta y es clave para edificar un gobierno que realmente gobierne. La alta dirección pública incluyendo la gestión de las juntas directivas de grupos interesados nicho que ha sido descuidado por la Nueva Gestión Pública y la Política Pública y se constituye como una de sus limitaciones teóricas y prácticas.

En esta parte final se presentan tres reflexiones con énfasis en los desafíos de la política pública del agua en México. La primera reflexión, es que la política pública del agua en México enfrenta el desafío de adaptar la nueva Ley General de Aguas al modelo de gestión integrada de recursos hídricos. Se requiere una ley que sea factible 
y que incorpore reglas e incentivos eficaces para dirigir las acciones de los actores y para lograr la integración. Dentro del nuevo diseño institucional es indispensable incentivar la conformación de alta gerencia e inclusión real de todos los actores involucrado, lograr la integración de la participación ciudadana y privada en las decisiones públicas. Para ello, la alta dirección gubernamental requiere líderes con nuevas capacidades y actitudes gerenciales, requieren dejar atrás las prácticas de centralización y abrirse a la nueva realidad.

La segunda reflexión final, se refiere a la necesidad de enfocarse en una correcta definición de problema de política pública. El propósito de este análisis fue identificar el valor que está ausente y el cual debe adoptar la nueva política federal del agua: la integración en la gestión integrada de los recursos hídricos. Además, es indispensable trabajar para encontrar el arreglo y diseño institucional que aumente la probabilidad de eficacia de los objetivos públicos. Para ello, la CONAGUA debe enfocarse en destinar recursos e incentivos a fortalecer las capacidades locales, sobre todo las capacidades gerenciales de los altos directivos de las organizaciones involucradas en la gestión del agua.

La última reflexión, es llamar la atención acerca de la adaptación y el aprendizaje en las políticas públicas. No hay que espantarse cuando una política comienza a desviarse de sus objetivos, sino que hay que estar atentos para ajustar y adaptar la política para regresarla a la ruta de los objetivos públicos, al núcleo duro de la definición del problema, ya que un mundo libre de restricciones sería completamente caótico e imprevisible (Majone, 1997). Las organizaciones pueden aprender y adaptarse a su medio sólo en la medida en que éste les presenta restricciones. En este sentido, el reto de la gestión y política federal del agua está en fortalecer la capacidad gerencial de las organizaciones locales para lograr la integración.

\section{BIBLIOGRAFÍA}

Aguilar, Luis F. (1993a) El estudio de las políticas públicas, México: ed. Porrúa.

----(1993b) Problemas públicos y Agenda de Gobierno, México: ed. Porrúa.

---(1993C) La hechura de las políticas, México: editorial Porrúa.

---(1993d) La implementación de las políticas, México: editorial Porrúa.

---(2006) Gobernanza y gestión pública, México: fondo de cultura económica.

---(2013a) Gobierno y Administración Pública, FCE-CONACULTA, México.

---(2013b) El gobierno del gobierno, INAP-México.

Bardach, Eugene (2000). “Problemas de la definición de problemas en el análisis de políticas”, en Luis F. Aguilar, Problemas públicos y agenda de gobierno (pp. 219-235), México D.F: Porrúa.

---- The implementation game: what happens after a bill becomes a law, Cambridge: MIT Press, 1977. DOI: 10.1177/106591297703000428.

Behn, Robert (1993), “El análisis de políticas y la política”, en Luis F. Aguilar (ed.) en La hechura de las políticas públicas, México: Porrúa.

Biswas, Asit (2008). Integrated Water Resources Management: Is It Working?, International Journal of Water Resources Development, 24:1, 5-22, DOI: 10.1080/07900620701871718

Cañez Cota, Antonio (2011). Patrones de decisión en la acción gubernamental municipal: el caso de Hermosillo, Sonora. Tesina de Maestría en Centro de Investigación y Docencia Económicas, México, D.F.

Casar María A. y Claudia Maldonado (2010), “Formación de agenda y proceso de toma de decisiones, una aproximación desde la Ciencia Política”, en Problemas, decisiones y soluciones: enfoques de política pública, México: CIDE-FCE.

Cabrero, Enrique (2005), “Capacidades institucionales en los gobiernos subnacionales en México”, en Revista Gestión y Política Pública, Vol. XII N003, México: CIDE.

Crozier, Michel y Friedberg, Erhard (1990), El actor y el sistema, las restricciones de la acción colectiva. México: Alianza Editorial Mexicana.

Comisión Nacional del Agua (CONAGUA) (2011), Buena gobernanza del agua para la gestión integrada de los recursos hídricos, Documento de posicionamiento, México, D.F: Comisión Nacional del Agua.

--- (2012) Atlas del Agua en México 2012, en: www.conagua.gob.mx 
--- (2012) Estadísticas del agua en México 2012 en: www.conagua.gob.mx

Constitución Política de los Estados Unidos Mexicanos, artículo cuarto párrafo sexto, consultado el 25 de septiembre de 2013 en: www.diputados.gob.mx

Diario Oficial de la Federación, decreto por el que se declara reformado el artículo cuarto constitucional de la Constitución Política de los Estados Unidos Mexicanos, 8 de febrero de 2012. Consultado el 25 de septiembre de 2013 en: http://dof.gob.mx/nota_detalle.php?codigo=5232952\&fecha=08/02/2012

Dictamen de la Ley General de Aguas (2015), Camara de Diputados, consultado el 15 de abril de 2015 en http:// www.cmdrs.gob.mx/comisiones/COTLEG/Documents/2015/3a_ordinaria/20150305-I1\%28ley-agua\%29.pdf

Domínguez Serrano, Judith (2006), La gobernanza del agua en México y el reto de la adaptación en zonas urbanas: el caso de la Ciudad de México, México, D.F: Anuario de estudios urbanos, UAM Azcapotzalco.

--- (2010). El acceso al agua y saneamiento: un problema de capacidad institucional local. Análisis en el estado de Veracruz, Gestión y Política Pública, Volumen XIX, número 2, II semestre de 2010, pp. 311-350.

Dror, Yezehkel (1983), Public Policymaking Reexamined, United States: Transaction Publishers. DOI: 10.1016/0099-3964(70)90022-0.

Edelenbos Jurian, Bressers Nanny and Scholten Peter (2013). Water governance as connective capacity, Burlington, VT: Ashgate. DOI: 10.3390/w5020640.

Heylighen, Francis (1999), "The growth of structural and funtional complexity during evolution" en F. Heylighen, J. Bollen \& A. Riegler (eds.) The Evolution of Complexity, Dordrecht: Klewer Academic, p. 17-44.

Kingdon, John (1984). Agendas, alternatives and public policies, Boston: Little, Brown \& Co. DOI: 10.1017/ s0143814×00003068.

Lasswell, Harold (1993), “La orientación hacia las políticas”, en Luis F. Aguilar (ed.) El estudio de las políticas públicas, México: Editorial Porrúa.

Lindblom, Charles E. (1993) “La ciencia de salir del paso" en Luis F. Aguilar Villanueva (ed.) La hechura de las políticas, México: Editorial Porrúa.

Linders, Stephen y Peters, Guy (1993), "Instrumentos de gobierno: percepciones y contextos", en Revista Gestión y Política Pública: 2, México: CIDE.

Majone, Giandomenico (2009), “Agenda Setting” DOI: 10.1093/oxfordhb/9780199548453.003.0011, en The Oxford Handbook of Public Policy, Oxford: Oxford University Press. DOI: 10.1093/oxfordhb/9780199548453.001.0001.

----- (1997) Evidencia, argumentación y persuasión en la formulación de políticas, México, D.F: Fondo de Cultura Económica.

Merino, Mauricio (2010), "La importancia de la ética," en Merino, Mauricio (editor): Problemas, decisiones y soluciones, enfoques de política pública, México: CIDE-FCE.

----- (2009) "Sobre la Evaluación de Políticas Públicas", texto de la Conferencia dictada en la Auditoría Superior de la Federación, México, D.F., abril 2009.

Morse, Kristin y Raymond Struyk (2006), Policy Analysis for effective development, sterngthening transition economies, United States: Lynne Rienner.

Nava, Luzma y Sandoval, Samuel (2014). Multi-tiered Governance of the Rio Grande/Bravo Basin: The Fragmented Water Resources Management Model of the United States and Mexico. International Journal of Water Governance (2), pp. 85-106. DOI: 10.7564/13-IJWG23.

Organización de las Naciones Unidas (2012), Status report on the application of the integrated approaches to water resources management, Nairobi: United Nations Environment Programme.

--- (2008). Status Report on Integrated Water Resources Management and Water Efficiency Plans, consultado el 9 de abril de 2015 en: http://www.unwater.org/downloads/UNW_Status_Report_IWRM.pdf

Organización para la Cooperación y Desarrollo Económico (OCDE) (2012), Hacer posible la reforma de la gestión del agua en México: diagnóstico y propuestas, México: OCDE.

Parsons, Wayne (2007), Políticas públicas: una introducción a la teoría y la práctica del análisis de políticas públicas, México: FLACSO. 
Pressman, Jeffrey y Wildavsky, Aaron (1984), Implementation: How Great Expectations in Washington Are Dashed in Oakland, United States: University of California Press.

Programa Nacional Hídrico (2014). Comisión Nacional del Agua, consultado el 30 de abril del 2015 en: http:/l dof.gob.mx/nota_detalle.php?codigo $=5339732 \&$ fecha $=08 / 04 / 2014$

Reglamento de la Ley de Aguas Nacionales (2015) (Última reforma publicada en el Diario Oficial de la Federación el 24-05-2011), consultada el 9 de abril de 2015 en: http://www.conagua.gob.mx/Contenido.aspx?n1=2\&n2=14

Ruano de la fuente, Jose Manuel (2010). Contra la participación: discurso y realidad de las experiencias de participación ciudadana, Política y Sociedad, 2010, Vol. 47 Núm. 3: 93-108.

Secretaría de Medio Ambiente y Recursos Naturales (SEMARNAT) (2012), Informe de la situación del medio ambiente en México, compendio de estadísticas ambientales, indicadores clave y de desempeño ambiental, México: SEMARNAT.

Torregrosa, Maria Luisa, et. al. (2012) Los recursos hidricos en Mexico en Blanca Jimenez y Jose Galizia (coord.) Diagnostico de las Americas, Foro Consultivo Científico y Tecnológico, AC, Mexico.

Transparencia Internacional (2008), Global corruption report, corruption in the water sector, United States: Cambridge University Press.

Weimer, A. y Aidan Vining (2002), Policy Analysis: concepts and practice, 5 edition, United States of America: Pearson. 